\title{
Remark on my paper: On Skolem's theorem.
}

\author{
By Gaisi Takeuti
}

(Received Dec. 10, 1956)

It was proved in my paper [1] that if the system of axioms of Fraenkel-von Neumann of the set-theory is consistent, then system remains consistent after addition of the axiom that every set is a univalent image of $\omega$. This result was established by Theorems 1, 2 of that paper. I should like to remark now that from the proof of these theorems follows immediately also the following result:

"Let $\Gamma_{1}$ be any consistent system of axioms in Gentzen's $L K$, representing mathematically a certain domain $D$ of elements. Then $\Gamma_{1}$ remains consistent after addition of the system of axioms of the theory of natural numbers, and the axiom that every element of $D$ is a univalent image of a natural number.

I shall formulate this result more precisely in the following lines, and indicate how to prove it.

We begin with $\Gamma_{a}$, the system of axioms of "arithmetic" consisting of axioms of the theory of natural numbers except the axiom of mathematical induction. In this paper, $\Gamma_{a}$ means the following axioms :

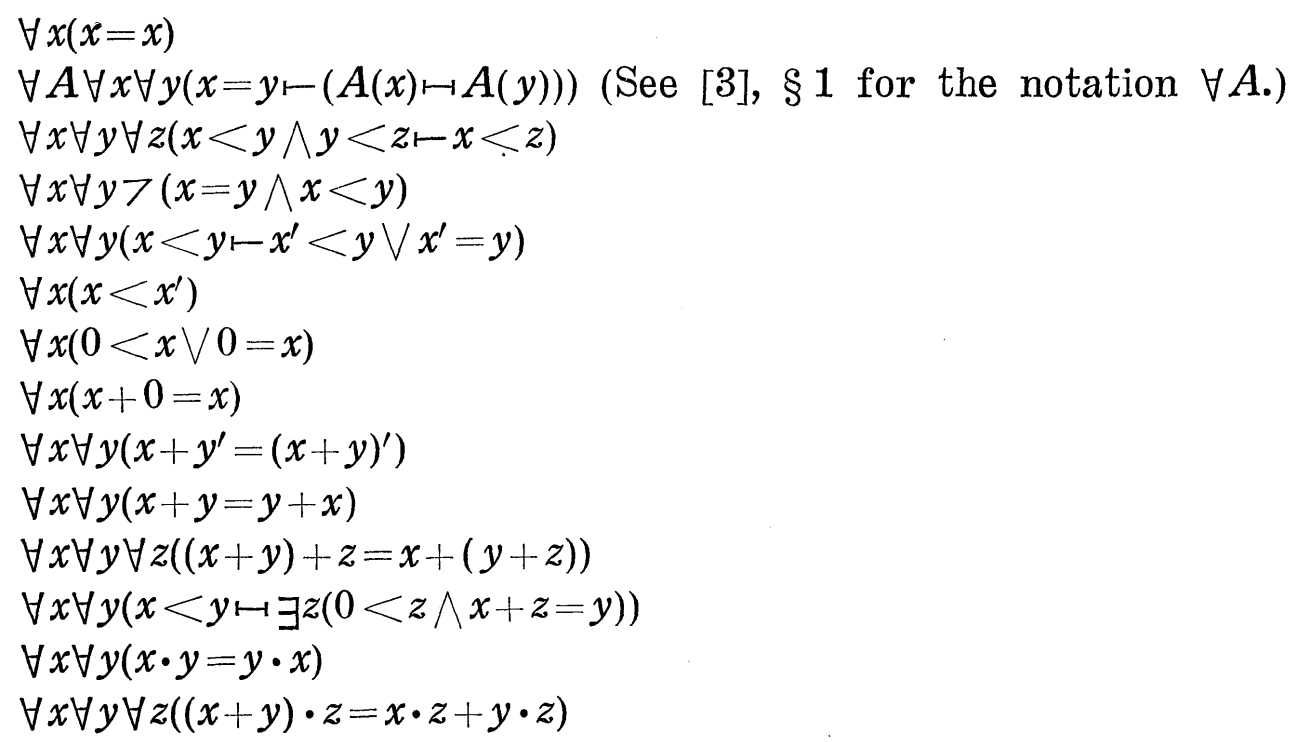


$\forall x\left(x \cdot 0^{\prime}=x\right)$

$\forall x \forall y \forall z((x \cdot y) \cdot z=x \cdot(y \cdot z))$

$\forall x\left(j\left(g_{1}(x), g_{2}(x)\right)=x\right)$

$\forall x \forall y\left(g_{1}(j(x, y))=x \wedge g_{2}(j(x, y))=y\right)$

$\forall x\left(0<x \vdash g_{2}(x)<x\right)$

$\forall x\left(0^{\prime}<x \vdash g_{1}(x)<x\right)$

$\forall x \forall y\left(y<x \vdash j(x, y)=x^{2}+y\right)$

$\forall x \forall y\left(x \leqq y \vdash j(x, y)=y^{2}+y+x\right)$

Then, we have the following theorem.

THEOREM. Let $\Gamma_{1}$ be consistent axioms in LK and satisfy the equality axioms with regard to $=$. (See [1] for equality axioms) Moreover, we assume that none of special variables, functions and predicates other than $=$ is contained in $\Gamma_{1}$ and $\Gamma_{a}$ at the same time. Then the following axioms are consistent in $L K$.

$\Gamma_{1}{ }^{e_{1}()} \quad$ (See [2], $\S 7$ for the notation $\left.\Gamma_{1}{ }^{{ }_{1}()}\right)$ where $e_{1}()$ is a predicate not contained in $\Gamma_{1}$ nor $\Gamma_{a}$.

$e_{1}(s)$ for every special variable $s$ in $\Gamma_{1}$.

$\forall x_{1} \cdots \forall x_{k} e_{1}\left(f\left(x_{1}, \cdots, x_{k}\right)\right)$ for every function $f$ in $\Gamma_{1}$.

$\forall x(x=x)$

$\forall A \forall x \forall y(x=y \vdash(A(x) \mapsto A(y)))$

$\forall x>\left(e_{1}(x) \wedge n(x)\right)$, where $n()$ is a predicate not contained in $\Gamma_{1}$ nor $\Gamma_{a}$. $n(0)$

$\forall x\left(n(x) \vdash n\left(x^{\prime}\right) \wedge n\left(g_{1}(x)\right) \wedge n\left(g_{2}(x)\right)\right)$

$\forall x \forall y(n(x) \wedge n(y) \vdash n(x+y) \wedge n(x \cdot y) \wedge n(j(x, y)))$

$\Gamma_{a}^{n()}$

$\forall A \forall x\left(A(0) \wedge \forall x\left(A(x) \vdash A\left(x^{\prime}\right)\right) \wedge n(x) \vdash A(x)\right)$

$\forall x \exists y\left(n(y) \wedge x=f_{0}(y)\right)$, where $f_{0}$ is a funcion not contained in $\Gamma_{1}$ nor $\Gamma_{a}$.

For the proof of this theorem we use the following three lemmas.

LEMMA 1. Let $\Gamma_{1}$ and $\Gamma_{2}$ be two consistent systems of axioms and let $\Gamma_{i}(i=1,2)$ satisfy the equality axioms with regard to $\stackrel{i}{=}$. Moreover, we assume that none of special variables, functions and predicates is contained in $\Gamma_{1}$ and $\Gamma_{2}$ at the same time. Let $e_{1}\left(\right.$ ), $e_{2}($ ) be two predicates not contained in $\Gamma_{1}$ nor $\Gamma_{\mathrm{2}}$. Then the following system of axioms $\tilde{\Gamma}$ is consistent.

$$
\begin{aligned}
& \Gamma_{1}{ }^{e_{1}()} \\
& \Gamma_{2}{ }^{e_{2}()} \\
& e_{1}\left(s^{1}\right) \text { for every special variable } s^{1} \text { contained in } \Gamma_{1} \text {. }
\end{aligned}
$$


$e_{2}\left(s^{2}\right)$ for every special variable $s^{2}$ contained in $\Gamma_{2}$. $\forall x_{1} \cdots \forall x_{k} e_{1}\left(f^{1}\left(x_{1}, \cdots, x_{k}\right)\right)$ for every function $f^{1}$ contained in $\Gamma_{1}$. $\forall x_{1} \cdots \forall x_{k} e_{2}\left(f^{2}\left(x_{1}, \cdots, x_{k}\right)\right)$ for every function $f^{2}$ contained in $\Gamma_{2}$. $\forall x_{1} \cdots \forall x_{k}\left(e_{2}\left(x_{1}\right) \bigvee \cdots \vee e_{2}\left(x_{k}\right) \vdash f^{1}\left(x_{1}, \cdots, x_{k}\right)=s^{1}{ }_{0}\right)$ for every function $f^{1}$ contained in $\Gamma_{1}$, where $s_{0}^{1}$ is a fixed special variable contained in $\Gamma_{1}$. $\forall x_{1} \cdots \forall x_{k}\left(e_{1}\left(x_{1}\right) \bigvee \cdots \vee e_{1}\left(x_{k}\right) \vdash f^{2}\left(x_{1}, \cdots, x_{k}\right)=s^{2}{ }_{0}\right)$ for every function $f^{2}$ contained in $\Gamma_{2}$, where $s^{2}{ }_{0}$ is a fixed special variable contained in $\Gamma_{0}$.

$\forall x_{1} \cdots \forall x_{i}\left(p^{1}\left(x_{1}, \cdots, x_{i}\right) \vdash e_{1}\left(x_{1}\right) \wedge \cdots \wedge e_{1}\left(x_{i}\right)\right)$ for every predicate $p^{1}$ contained in $\Gamma_{1}$.

$\forall x_{1} \cdots \forall x_{\imath}\left(p^{2}\left(x_{1}, \cdots, x_{i}\right) \mapsto e_{2}\left(x_{1}\right) \wedge \cdots \wedge e_{2}\left(x_{i}\right)\right)$ for every predicate $p^{2}$ contained in $\Gamma_{2}$.

$\forall x\left(e_{1}(x) \vee e_{2}(x)\right)$

$\forall x>\left(e_{1}(x) \wedge e_{2}(x)\right)$.

LEMMA 2. Under the same hypothesis as in Lemma 1, $\tilde{\Gamma}$ satisfies the equality axioms with regard to $=$, provided that $a=b$ is defined to be $\left(e_{1}(a) \wedge e_{1}(b) \wedge a \stackrel{1}{=} b\right) \vee\left(e_{2}(a) \wedge e_{2}(b) \wedge a \stackrel{2}{=} b\right)$.

LEMMA 3. $\Gamma_{a}$ is consistent in $L K$.

We need not dwell upon the proof of these lemmas which are immediate. Our Theorem is deduced as follows.

In virtue of Lemma 3, $\Gamma_{a}$ can be used as $\Gamma_{2}$ in Lemma 1; we use $\Gamma_{1}$ in our Theorem as $\Gamma_{1}$ in Lemma 1. Then we can follow, in virtue of Lemmas 1, 2, the proof of Theorems 1, 2 in [1] in regarding $\Gamma_{0}$ in [1] as $\tilde{\Gamma}$ and $e()$ in [1] as $e_{2}($ ). We obtain thus our Theorem, in considering $n(a)$ in [1] as $n(a)$ in our Theorem.

\section{References}

[1] G. Takeuti: On Skolem's theorem. J. Math. Soc. Japan, 9 (1957).

[2] : On a generalized logic calculus. Jap. J. Math., 23 (1953), pp. 39-96; Errata to 'On a generalized logic calculus'. Jap. J. Math., 24 (1954), pp. 149-156.

[3] : A metamathematical theorem on functions. J. Math. Soc. Japan, 8 (1956) pp. 65-78. 